This is the submitted version of the following article: Pina-Estany, J. et al. "A statistical analysis of nanocavities replication applied to infection moulding" in Int. communications in heat and mass transfer (Ed. Elsevier), vol. 81 (2017), p. 131-140.

๑) 2017. This manuscript version is made available under the CC-BY-NC-ND 4.0 license http://creativecommons.org/licenses/bync-nd/4.0/

\title{
A statistical analysis of nanocavities replication applied to injection moulding
}

\author{
J.Pina-Estany ${ }^{1} ;$ C.Colominas ${ }^{2} ;$ J.Fraxedas ${ }^{3} ;$ J.Llobet $^{4} ;$ \\ F.Perez-Murano ${ }^{4}$; J.M.Puigoriol-Forcada ${ }^{1}$; D.Ruso ${ }^{3}$, present address $^{2}$; A.A.Garcia-Granada ${ }^{1}$; \\ Corresponding author: J.Pina-Estany, jordi.pina@iqs.edu \\ ${ }^{1}$ IQS-Universitat Ramon Llull, Via Augusta, 390. 08017, Barcelona (Spain); \\ ${ }^{2}$ Flubetech SL, Carrer Montsià, 23. 08211, Castellar del Vallès (Spain); \\ ${ }^{3}$ Catalan Institute of Nanoscience and Nanotechnology (ICN2), CSIC and The Barcelona Institute of Science and Technology, Campus \\ UAB, Bellaterra. 08193, Barcelona (Spain); \\ ${ }^{4}$ Institut de Microelectrònica de Barcelona (IMB-CNM, CSIC), C/Til.lers, Campus Universitat Autònoma de Barcelona. Cerdanyola del \\ Vallès. Barcelona (Spain)
}

\begin{abstract}
The purpose of this paper is to investigate both theoretically and experimentally how nanocavities are replicated in the injection moulding manufacturing process. The objective is to obtain a methodology for efficiently replicate nanocavities.

From the theoretical point of view, simulations are carried out using a submodeling approach combining Solidworks Plastics for a first macrosimulation and Fluent solver for a subsequent nanosimulation. The effect of the four main factors (melt temperature, mould temperature, filling time and cavity geometry) are quantified using an statistical $2^{4}$ factorial experiment. It is found that the main effects are the cavity length, the mould temperature and the polymer temperature, with standardized effects of 5, 3 and 2.6, respectively. Filling time has a negative 1.3 standardized effect.

From the experimental point of view, Focused Ion Beam technique is used for mechanizing nanocavities in a steel mould. The replication achieved in polycarbonate injection is quantified using an Atomic Force Microscope. It is observed how both the geometry and the position of the cavities in the mould affect its replication.
\end{abstract}

Keywords: nanoscale, simulation, AFM, heat transfer, injection moulding, CFD, submodeling

\section{Introduction ${ }^{1}$}

The demand of products with nanostructured surfaces is increasing due to the wide field of applications. The applications cover the medical and pharmaceutical industry with lab-on-chips devices (Oh [1]) and surfaces with selfcleaning (Yoo [2]) and antimicrobial surfaces (Kim [3]); environment applications to purificate water (Baruah [4]) and air and to remediate the soil; optical applications for achieving antireflection (Christiansen [5]), transmission and contrast enhancements, etc.

Injection moulding is a manufacturing process with a high cost of entry but a low production cost (Fagade [6]). Therefore, it is a promising process for obtaining large series of plastic parts with nanostructured surfaces. However, achieving a good replication is not trivial because the obtained replication index depends on many factors, among them process factors (mould and polymer temperatures, filling time, holding pressures, viscosity of the polymer, etc.) and geometrical factors (depth, wide, aspect ratio of the cavity, etc.). The purpose of this paper is to give light on how -and how much- these factors affect the

\footnotetext{
19bbreviations: AFM=Atomic Force Microscope. $\mathrm{CFD}=$ Computational Fluid Dynamics. FIB=Focused Ion Beam. $\mathrm{SEM}=$ Scanning Electron Microscopy. $\mathrm{VOF}=$ Volume of Fluid.
}

replication and, hence, avoid failures like the one showed in the figure 1 , where we show how a nanocavity was not successfully replicated by the polymer.

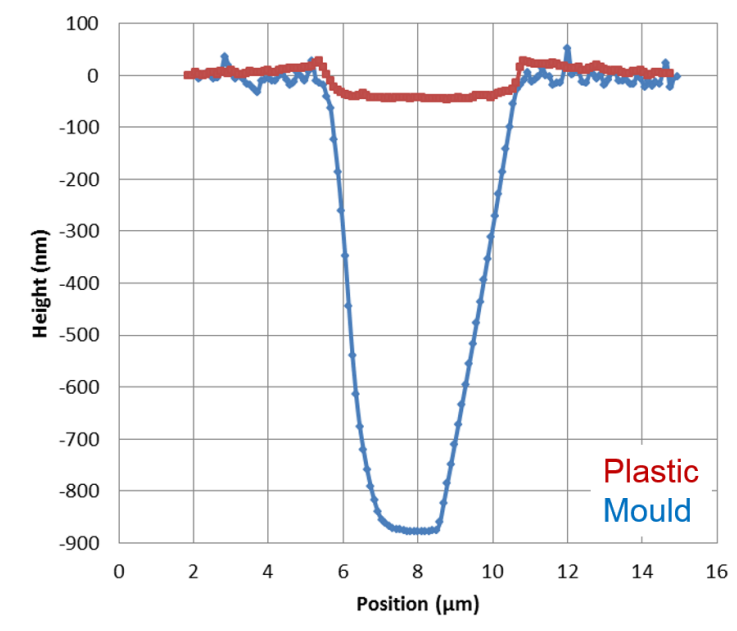

Figure 1: AFM measurement of a failed attempt to fill a nanocavity. Even though the cavity is $900 \mathrm{~nm}$ depth, the polymer penetrates only $50 \mathrm{~nm}$. The reason is that, due to the big area/volume ratio of the nanoworld, the polymer reaches its no flow temperature far before reaching the end of the cavity. This paper aims to give light into how the process and geometrical parameters should be adjusted in order to improve the replication. 
The study is done both from a theoretical point of view using computer fluid dynamics but also from an experimental point of view.

\section{Material and methods}

This section is divided in two subsections, the first one referring to the simulation setup (subsection 2.1) and the second one related to the experimental setup (subsection $2.2)$.

\subsection{Simulation setup}

When speaking about simulation of fluids dynamics at the nanoscale, the first question that arises is whether the continuum fluid approach is valid for such scale or not. The small cavities we simulate and experimentally fill with polymer are of around $2 \mu \mathrm{m} \cdot 10 \mu \mathrm{m} \cdot 200 \mathrm{~nm}=4 \cdot 10^{-18} \mathrm{~m}^{3}$, using the polycarbonate density $\left(1200 \mathrm{~kg} / \mathrm{m}^{3}\right)$ and its molar mass $(254.3 \mathrm{~g} / \mathrm{mol})$ we calculate that each cavity is filled with around $4 \cdot 10^{11}$ polycarbonate units. Because of this elevated number of polycarbonate units in each cell and because the filled cavities are larger than 10 molecular diameters, which is, according to the literature (Eijkel [7]), the threshold where deviations from the classical continuum theory are observed due to the quantisation of molecular layers, the continuum approach is considered valid for this application. In consequence, the following calculations are carried using the conventional Computational Fluid Dynamics approach and the Finite Volume Method for discretizing the Navier-Stokes equations.

The first problem that arises when aiming to simulate how a polymer flows and fills a mould with a nanostructured pattern is the meshing problem. Meshing the whole mould with a nanoscale mesh would lead to a computationally unaffordable calculation, as a fast exercise: a part of $(50 \cdot 50 \cdot 3) \mathrm{mm}^{3}$ meshed with hexahedral mesh of $1 \mathrm{~mm}$ has up to 7500 elements and its calculation takes in a personal computer (Intel(R) Core(TM) i5-3320M CPU @ 2.60 $\mathrm{GHz}$ ) around 600s. If the model is meshed with quadrilateral elements of $1 \mathrm{~nm}$ per vertex we have instead of 7500 elements up to $7.5 \cdot 10^{30}$ elements, what leads to a calculation time of $1.9 \cdot 10^{22}$ years without even taking into account the time step reduction necessary for maintaining the Courant number below a reasonable margin. And, apart from the time problem, no computer has enough memory for handling this huge amount of elements. A logical solution to this problem seems to be to refine the mesh around the cavities, but this leads to unphysical results due to the too big ratio of scales as already stated in the literature (Tofteberg, [8], Yu [9]). We worked around this problem by using a submodeling approach.

The submodeling approach -multiscale simulation according to some authors- consists in carrying out two simulations consecutively. The first is a macro simulation carried out using Solidworks Plastics and the second is a nanoscale simulation carried out using CFD. In order to link the two simulations, the boundary conditions are transferred from the first to the second simulation:

1. Macro simulation first stage: A first conventional simulation of mould filling is carried out in the geometry shown in the figure 2. The simulations are done in this geometry because it is the geometry that was used for manufacturing the mould used for the Experimental results section.

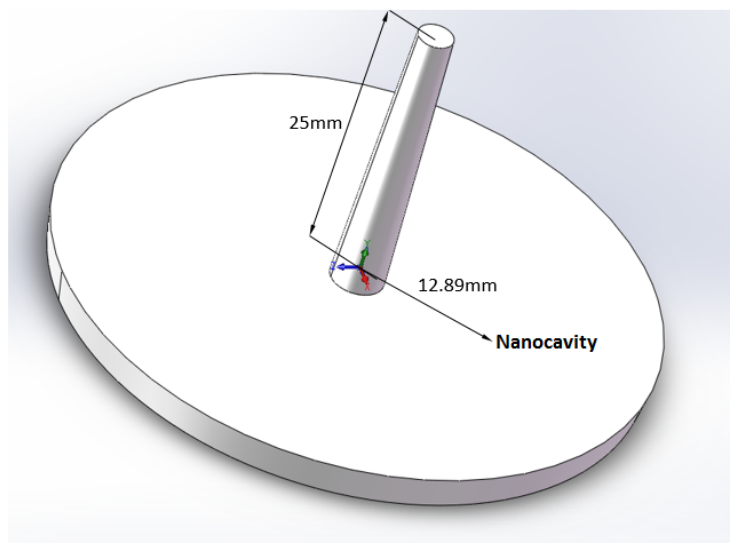

Figure 2: CAD model used for the macro simulation first stage and corresponding to the part manufactured using injection moulding as exposed in the Experimental setup section.

In the first stage it is considered that the fluid goes through the cavity without noticing its presence (see figure 3). The reason is that the mark is so small that the polymer goes through it with around $0.15 \mathrm{~ms}$ for a normal injection. From this macro simulation we extract the pressure profile and the melt front temperature of the polymer when it contacts for the first time the cavity. Furthermore, due to the small size of the nanocavity it is also considered that the cavity presence does not affect the bulk flow.

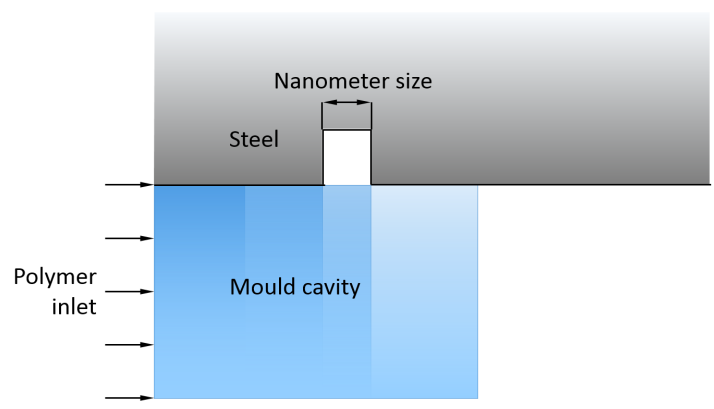

Figure 3: Macro simulation, the graduates tones in blue indicate the polymer advance. It is considered that the fluid goes through the cavity without noticing its presence.

2. Nano simulation: Once the polymer has gone through the mark, the pressure starts pushing the polymer into the mark (see figure 4). This stage gets to an end when the polymer solidifies due to the heat transfer with the walls. 


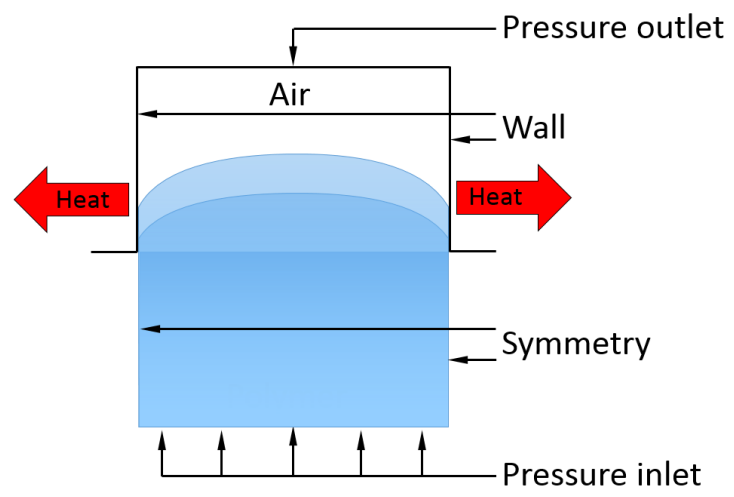

Figure 4: Nano simulation, the graduated tones in blue indicate the polymer advancing into the nanocavity until it reaches the glass transition temperature. The boundary conditions are indicated.

The nanosimulation is stopped when the polymer reaches its glass transition temperature $\left(T_{g}\right)$ in all the region inside the cavity.

Next, we expose the nanosimulation setup:

\subsubsection{Models}

The models used are the volume of fluid model, the energy and the solidification/melting model.

The VOF method tracks the interface evolution with an Eulerian approach, i.e., without modifying the mesh during the calculation. The explicit approach is used in order to activate the Geometric Reconstruction scheme and obtain hence a sharper interface. Laminar flow is considered due to the low Reynolds number.

The energy model is used with the purpose to account for the heat transfer between the polymer and the walls.

The solidification and melting model is used in order to model the solidification of the fluid due to the heat transfer with the walls.

\subsubsection{Mesh}

A nearly structured quadrilateral mesh of 70000 elements is used.

\subsubsection{Materials}

Three materials are involved in the simulation: polymer, air and steel:

- Polymer:

- Density $=1200 \mathrm{~kg} / \mathrm{m}^{3}$. Density dependance of the fluid with the temperature and pressure is not taken into account for simplification purposes. When considering the error introduced by making this simplification, we identify in the PVT diagram that, if the density is taken as the average between the initial and the final conditions, the introduced error is less than a $5 \%$.

\section{- Viscosity}

The viscosity of the polymer is modeled using the Cross-WLF model. The Cross-WLF models the dependance of the polymer with the temperature (viscosity inversely proportional to temperature) and with the shear rate (viscosity inversely proportional to shear rate). For the polycarbonate, the viscosity vs. shear rate for different temperatures is shown in the figure 5 .

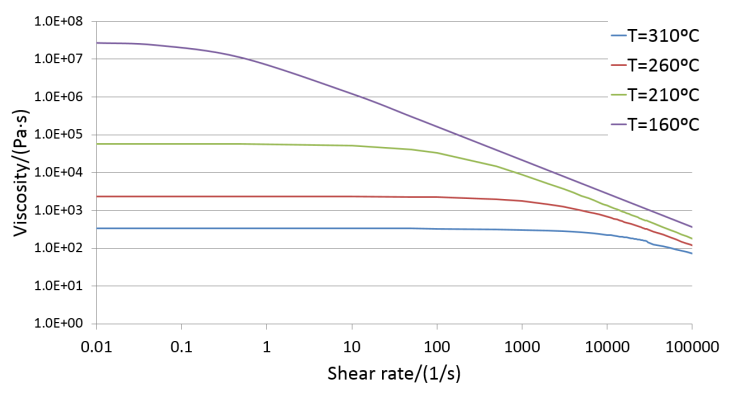

Figure 5: Cross-WLF model for modeling viscosity as a function of temperature and shear rate for polycarbonate.

The equations modeling the Cross-WLF are:

$$
\begin{gathered}
\eta(T, \dot{\gamma})=\frac{\eta_{0}(T)}{1+\frac{\eta_{0}(T) \dot{\gamma}}{\tau^{*}}} \\
\eta_{0}(T)=D_{1} e^{\frac{-A_{1}\left(T-D_{2}\right)}{A_{2}+\left(T-D_{2}\right)}}
\end{gathered}
$$

Where $\eta$ is the melt viscosity of the polymer, $\eta_{0}$ is the zero shear viscosity or the Newtonian limit in which the viscosity approaches a constant at very low shear rates, $\dot{\gamma}$ the shear rate, $T$ is the temperature of the polymer, $A_{1}, A_{2}, D_{1}$, $D_{2}, n$ and $\tau^{*}$ are constants of the table 1 (Osswald [11]). $A_{1}, A_{2}, D_{1}$ and $D_{2}$ are data-fitted coefficients, $n$ is the power law index in the high shear rate regime, determined by curve fitting and $\tau^{*}$ is the critical stress level at the transition to shear thinning, determined by curve fitting.

Table 1: Cross-WLF constants for PC
\begin{tabular}{|l|l|l|}
\hline Constant & Value & Unit \\
\hline$A_{1}$ & 8.4 & - \\
\hline$A_{2}$ & 246.8 & $\mathrm{~K}$ \\
\hline$D_{1}$ & 462 & $\mathrm{~Pa} \mathrm{~s}$ \\
\hline$D_{2}$ & 573 & $\mathrm{~Pa} \mathrm{~s}$ \\
\hline$\tau^{*}$ & 8437056 & $\mathrm{~Pa}$ \\
\hline$n$ & 0.116 & - \\
\hline
\end{tabular}

- Heat capacity $=1700 \mathrm{~J} / \mathrm{kg} / \mathrm{K}$

- Thermal conductivity $=0.173 \mathrm{~W} / \mathrm{m} / \mathrm{K}$ 
- Pure solvent melting heat $=105 \mathrm{KJ} / \mathrm{kg}$

- Solidus and Liquidus Temperature $=420 \mathrm{~K}$

- Air

- Incompressible air approach is used.

- Viscosity.

The viscosity of the air is artificially increased to $0.1 \mathrm{~Pa} \cdot \mathrm{s}$ instead of its value of $1.8 \cdot 10^{-5} \mathrm{~Pa} \cdot \mathrm{s}$, the reason is that high ratio of viscosity between phases leads to divergences. This strategy is also used by Tofteberg [8].

- Steel. Although in the experimental part a diamondlike carbon coating is used, the thickness of the coating is insignificant and, hence, not taken into account in the simulation parameters.

\subsubsection{Boundary conditions}

- Inlet: A transient pressure inlet is used via a Profile function. The profile is extracted from the macro simulation in the node where the nanocavity is. Due to the small size of the nanocavity, the pressure profile is considered constant through all the inlet.

- Walls: No shear rate wall is used. Reason is that using no-slip boundary condition the simulation leads to unphysical results due to the contact line discontinuity of the Navier-Stokes equation, what is already reported in the literature (Gal [12]) and specifically for flows at the nanometre scale (Eijkel [7]). Apart from the theory, Kuhn [13] publishes AFM sections of different short shots at different mold temperatures of molded v-grooves and it is clearly seen that the contact line went into the grooves, hence, slipping through the wall.

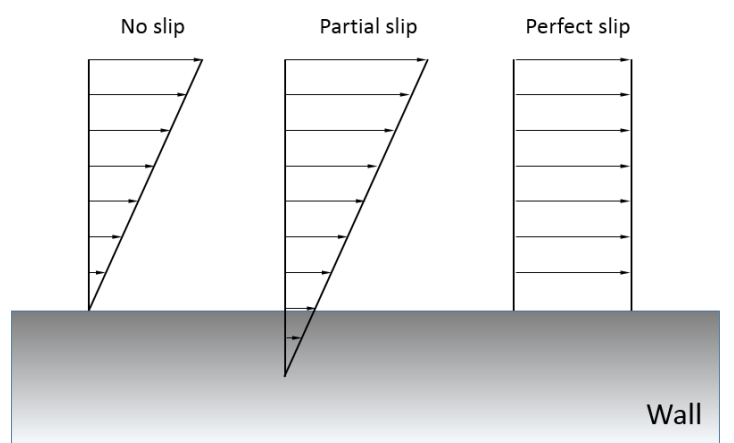

Figure 6: Different approaches for fluid velocity at the wall. A perfect slip approach was used for the walls in order to avoid the unphysical results due to the contact line discontinuity of the Navier-Stokes equation.

- Outlet: 1bar absolute pressure outlet is used. This approach means that the compression of the gas due to the trapping of the air is not taken into account, if the air pressure is calculated using ideal gas law we obtain $P=P_{0} /(1-x / L)$, where $x$ is the filled depth of the cavity and $L$ the total depth of the cavity. This equation, once plotted is shown in figure 7 .

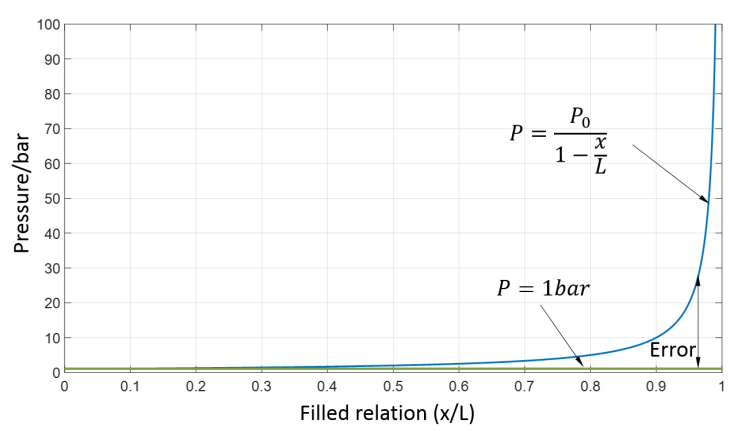

Figure 7: Pressure that would be generated inside the cavity due to compression of the air (blue) and 1 bar pressure considered in the simulations with pressure outlet boundary condition (green).

It is observed that our approach has an error minor than $10 \%$ if the cavity is filled less than its $10 \%$. When more than this percentage is filled, the simulation will overestimate the filling of the cavity, because the calculation would not take into account the opposition done by the air.

Future work will be the implementation of a compressible air material and the consideration of a real wall instead of a pressure outlet. From an industrial point of view, since air trap is an effecting factor, injection mould vents should be considered in the design of the mould. Another way to vent a mould is the use of a mould vacuum. This is a very expensive option; however, sometimes it is the only option. This is achieved by sealing all parts of the mould cavity by the use of an O-ring. Once the mould is closed, all of the air is drawn out of the cavity before or at the same time the injection phase of the injection moulding cycle is started. With no air present in the cavity, a perfect condition has been created for trouble-free filling of the cavity (Toth Mold/Die Inc.).

- Symmetry: Symmetry is used in order to take into account that there is more fluid at the left and the right of the computationally domain of where we put the fluid (see figure 4). The symmetry boundary condition states zero normal velocity at the symmetry line and zero normal gradients of all variables at the symmetry line.

\subsubsection{Initial conditions}

The lower region is patched with a $\mathrm{VOF}=1$ of polymer, upper region is patched to be air at the same temperature of the walls. Reason for setting the pressure inlet away of the cavity beginning is to take into account the pressure loss of the polymer when getting into the cavity (Tofteberg [8]).

Initially, zero velocity at all the model is considered. 


\subsubsection{Design of experiments}

A four factors and two levels factorial experiment is carried out. The temperature of the melt, the temperature of the mould and the filling time are process factors, wherease cavity length is a size factor. The levels are shown in table 2 .

Table 2: Factorial design

\begin{tabular}{|l|l|l|}
\hline Factor & Low value & High value \\
\hline $\mathrm{T}_{\text {melt }}$ & $250^{\circ} \mathrm{C}$ & $310^{\circ} \mathrm{C}$ \\
\hline $\mathrm{T}_{\text {mould }}$ & $70^{\circ} \mathrm{C}$ & $100^{\circ} \mathrm{C}$ \\
\hline Filling time & $1 \mathrm{~s}$ & $3 \mathrm{~s}$ \\
\hline Cavity length (L) & $1000 \mathrm{~nm}$ & $2000 \mathrm{~nm}$ \\
\hline
\end{tabular}

It is important to state that the filling time determines the needed injection pressure. If the injection time to fill the cavity is small, it must have high injection speed, therefore, must have very high pressure. When the injection time is very long, the melt temperature decreases, viscosity increases, thus it increases the difficulty to fill and requires high injection pressure. There is a lowest point in the middle of the curve and this point is the best filling time which is corresponding to the lowest injection pressure (Fallis [14]). For the used filling times of $1 \mathrm{~s}$ and $3 \mathrm{~s}$ we see that $1 \mathrm{~s}$ needs more injection pressure than $3 \mathrm{~s}$, so the 3 s value is already at the left of the minimum filling time.

Concerning to the cavity length, a low level of 1000nm is used because even with this length, the injections with the low level of mould temperatures and the high level of filling time lead to a zero replication.

For the simulations, a maximum injection pressure of $134 \mathrm{MPa}$ is set, which is the maximum injection pressure of the Babyplast $6 / 10 \mathrm{p}$ with a $14 \mathrm{~mm}$ screw that IQS University has.

\subsection{Experimental setup}

A mould was specifically designed in order to explore different parameters on the plastic injection process, and facilitate the ulterior characterization of both, the mould and the injected plastic parts, by AFM and SEM. In the mould, specific micro/nano scale patterns have been fabricated with the goal of comparing the replication of such marks in the plastic parts with simulations described in the previous section. Next, we present a detailed description of the analysis performed on the mould and the plastic injection pieces.

\subsubsection{Design and fabrication of mould}

The mould was designed and fabricated in such a way that: (i) the mould can be easily disassembled, (ii) the surfaces in contact with the injected plastic are flat and can be measured with a commercial AFM instrument (in our case a Bruker ICON) and (iii) plastic can be injected with radial flow.

Mould was machined using coated carbide tools from modified AISI 420 steel named Mirrax 40 (by Uddeholm).
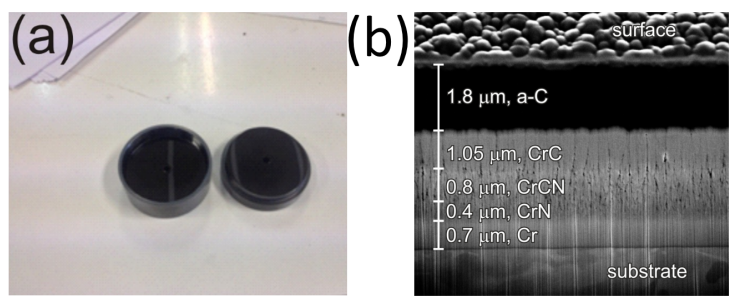

Figure 8: Mould after DLC coating: (a) picture, (b) image of a transversal section using FIB.

This steel is a pre-hardened $(40 \mathrm{HRC})$ re-melted stainless tool steel (composition: C $0.21 \%$, Si $0.9 \%$, Mn $0.45 \%$, Cr $13.5 \%$, Mo $0.2 \%$, Ni $0.6 \%, \mathrm{~V} 0.25 \%$ ). The mould was then hardened $\left(1020^{\circ} \mathrm{C}\right), 30$ minutes, gas quenched) and tempered $\left(450^{\circ} \mathrm{C}, 2 \mathrm{~h}\right)$ to a hardness value of $52 \mathrm{HRC}$.

Once the mould was fabricated, the critical surfaces were polished to optical quality finish. Upon polishing, the roughness decreased from approximately $\mathrm{Sq}=17 \mathrm{~nm}$ to $\mathrm{Sq}=3.5 \mathrm{~nm}$, as determined by AFM. This process was carried out using vibrational hand tools following several steps: (i) grinding stone 320-600 (ii) paper 500 (iii) acryl cloth, diamond fluid $15 \mu \mathrm{m}$ (iv) felt cloth, diamond fluid $3 \mu \mathrm{m}(\mathrm{v})$ cotton cloth, diamond fluid $1 \mu \mathrm{m}$.

\subsubsection{DLC coating of Mould}

Moulds are usually coated in order to increase their durability and achieve longer lifetimes while reducing maintenance and operational costs. The polished mould was coated at Flubetech with a Diamond Like Carbon [DLC] layer at $170^{\circ} \mathrm{C}$ using a CemeCon CC800/9 magnetron sputtering Physical Vapour Deposition (PVD) unit from $\mathrm{Cr}$ and graphite targets. The overall coating thickness is $4.75 \mu \mathrm{m}$ consisting of a gradient adhesion multilayer $(\mathrm{Cr}+$ $\mathrm{CrN}+\mathrm{CrCN}+\mathrm{CrC}$ ) plus a final amorphous carbon (aC) layer of $1.8 \mu \mathrm{m}$, which exhibits a hydrogen content of approx. $18 \%$. The morphology and roughness of the surfaces were determined with SEM and AFM. The resulting Sq value $(\approx 8 \mathrm{~nm})$ is larger as compared to the uncoated surfaces $(\approx 3.5 \mathrm{~nm})$. In addition, the microhardness was determined (3000 HV), using a Fischerscope H100 dynamic microprobe apparatus with a conventional Vickers indenter (load of $10 \mathrm{mN}$ ).

\subsubsection{FIB marks on mould}

Once the mould was characterized after the DLC coating, different shapes were designed for patterning the surface of the mould by FIB milling. The idea behind this is to extract information about how different variables like roughness, orientation of the patterns, size, shape, etc. can affect and determine the pattern-transfer to the final piece in the injection process. The designed patterns (crosses, rectangles and triangles) are shown in the figure 9. The chosen selection was then placed at different distances from the center of the mould. In this way, apart from the effect of the size and shape, we can extract information about 


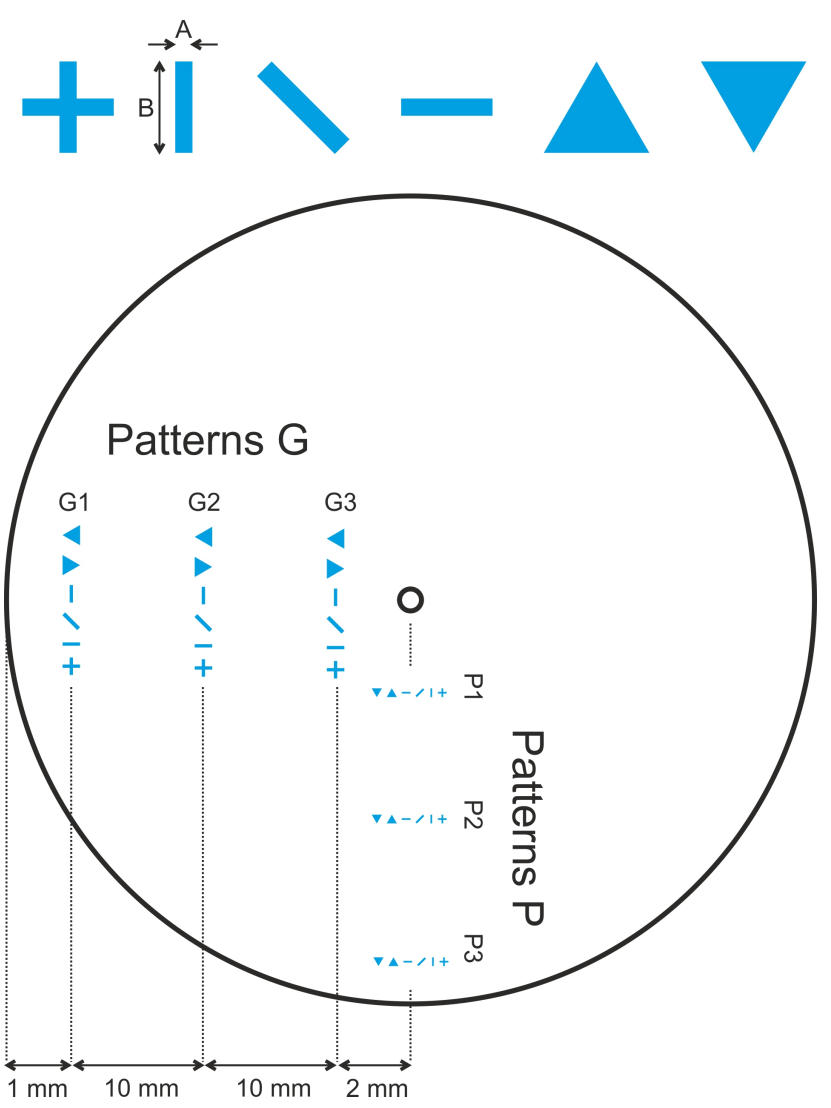

Figure 9: Designed FIB marks and their position for mould (not to scale). The diameter of the piece is $50 \mathrm{~mm}$ and the dimensions of patterns $\mathrm{G}$ and $\mathrm{P}$ are: $A=4 \mu \mathrm{m}, B=20 \mu \mathrm{m}$ and $A=2 \mu \mathrm{m}$ and $B=10 \mu \mathrm{m}$, respectively.
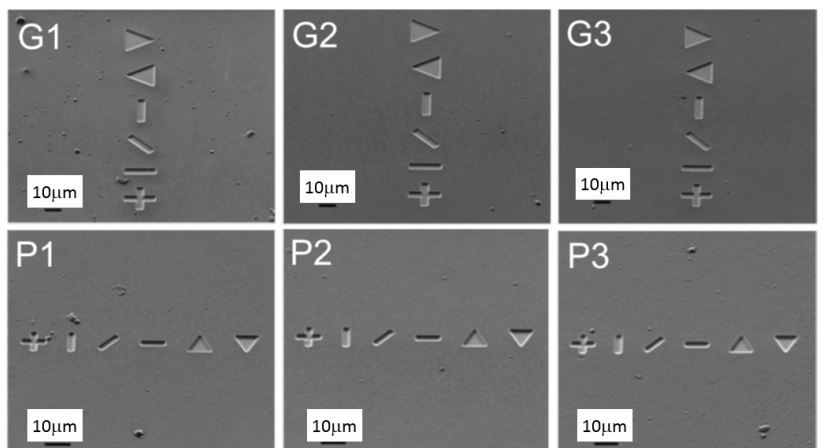

Figure 10: SEM images of the FIB patterns on mould, as described in figure 9 . how does the fluid flow-velocity affect the pattern transfer, an issue of great relevance in the injection process. A depth of $1 \mu \mathrm{m}$ was initially selected for all the marks, which was achieved with a $60.000 \mu \mathrm{As} / \mathrm{cm}^{2}$ dose determined from previous calibration tests on analogous DLC coatings on test samples of the same stainless steel. SEM images of the patterns on the mould at different locations, as indicated in figure 9, are shown in figure 10 . The depth of these marks was $875 \pm 25 \mathrm{~nm}$ as determined by AFM.

\subsection{Plastic injection using mould}

Microinjection moulding was performed using transparent polycarbonate (PC SABIC ${ }^{\circledR}$ LEXAN Resin 133R). Injection was performed in a Babyplast $610 \mathrm{P}$ plastic injection machine at a mould temperature of $90^{\circ} \mathrm{C}$ and injection pressure of 50 bar with the channel at $280^{\circ} \mathrm{C}$. The pressure was hold for 3 seconds in a first step and another 4 seconds for a second pressure of 70 bar.

\section{Results and discussion}

This section is divided in two subsections: one for the simulation results (subsection 3.1) and another one for the experimental results (subsection 3.2).

\subsection{Simulation results}

The replicated height $(\mathrm{H})$ for each simulation is measured as the height reached by the polymer at the center of the cavity when all the fluid inside the cavity is below its glass transition temperature, as it is shown in figure 11. It must be pointed out that some authors (Rytka [15] or Mannella [16]) consider that the polymer stops its movement not when its temperature is below the glass transition temperature but when is below its no flow temperature (NFT). The NFT does not have a standard method for measuring it and is considered a not well defined value. A common rule of thumb is to consider NFT $=T_{g}+30^{\circ} \mathrm{C}$. If we used this approach, all the heights would be slightly smaller.

The results for all the simulations are summarized in the table 3 .

The analysis of this $2^{4}$ factorial experiment leads to the Pareto diagram of figure 12. The effects are sorted from most significant to least significant and the length of each bar is proportional to the standardized effect, which equals the magnitude of the t-statistic that would be used to test the statistical significance of that effect. A vertical line is drawn at the location of the 0.05 critical value for Student's t. Any bars that extend to the right of that line indicate effects that are statistically significant at the $5 \%$ significance level.

The principal effects diagram is shown in figure 13 . The effects' results are summarized in table 4:

The factor with the biggest effect is the geometry of the mark. It is broadly shown in the literature that cavities with small aspect ratio are more difficult to fill, reason 


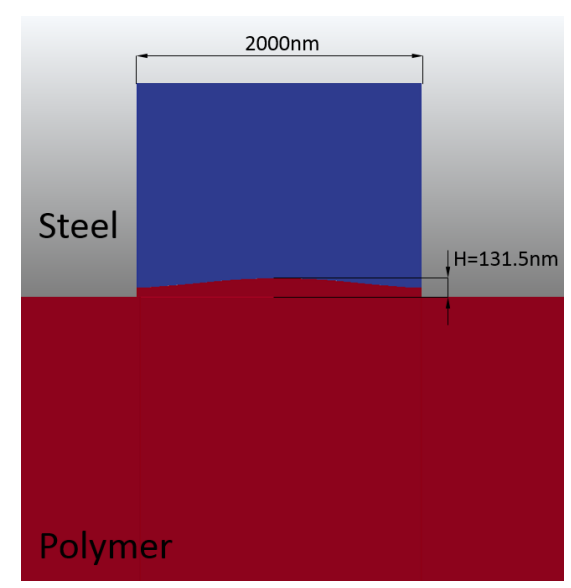

Figure 11: Image of the simulation with $\mathrm{T}_{\text {melt }}=250^{\circ} \mathrm{C}, \mathrm{T}_{\text {mould }}=$ $70^{\circ} \mathrm{C}$, fillingtime $=2 s$ and cavity length is $2000 \mathrm{~nm}$. The $\mathrm{H}$ measure is taken at the first time step where all the fluid inside the cavity is already below its glass transition temperature. Notice that the fluid progresion is smaller in the vicinity of the walls than in the center of the feature.

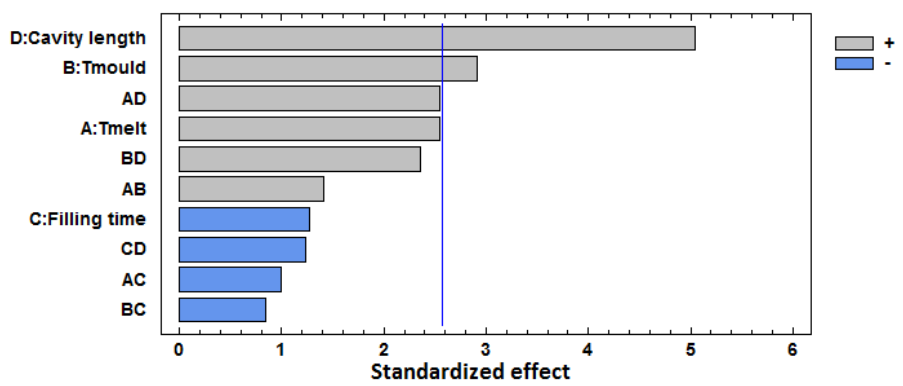

Figure 12: Pareto diagram. It is observed that the more representative factors are the cavity length and the mould temperature.

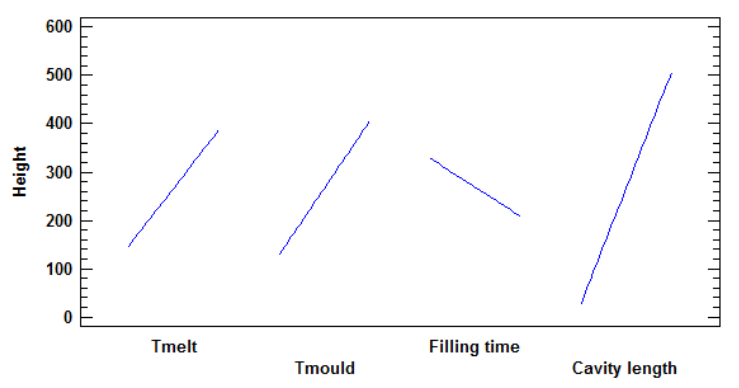

Figure 13: Principal effects diagram. It is seen that both the temperatures and the cavity length have a positive effect, in contrast to the filling time negative effect. The cavity length is the factor with a major impact in the cavities replication.
Table 3: Series of simulations

\begin{tabular}{|l|l|l|l|l|l|}
\hline & $\mathrm{T}_{\text {melt }} /{ }^{\circ} \mathrm{C}$ & $\mathrm{T}_{\text {mould }} /{ }^{\circ} \mathrm{C}$ & $\mathrm{t} / \mathrm{s}$ & $\mathrm{L} / \mathrm{nm}$ & $\mathrm{H} / \mathrm{nm}$ \\
\hline 1 & 250 & 70 & 1 & 1000 & 5.0 \\
\hline 2 & 310 & 70 & 1 & 1000 & 5.0 \\
\hline 3 & 250 & 100 & 1 & 1000 & 55.0 \\
\hline 4 & 310 & 100 & 1 & 1000 & 55.0 \\
\hline 5 & 250 & 70 & 3 & 1000 & 0.0 \\
\hline 6 & 310 & 70 & 3 & 1000 & 0.0 \\
\hline 7 & 250 & 100 & 3 & 1000 & 55.0 \\
\hline 8 & 310 & 100 & 3 & 1000 & 55.0 \\
\hline 9 & 250 & 70 & 1 & 2000 & 170.0 \\
\hline 10 & 310 & 70 & 1 & 2000 & 420.0 \\
\hline 11 & 250 & 100 & 1 & 2000 & 412.3 \\
\hline 12 & 310 & 100 & 1 & 2000 & 1500.0 \\
\hline 13 & 250 & 70 & 3 & 2000 & 131.5 \\
\hline 14 & 310 & 70 & 3 & 2000 & 308.3 \\
\hline 15 & 250 & 100 & 3 & 2000 & 350.7 \\
\hline 16 & 310 & 100 & 3 & 2000 & 762.5 \\
\hline
\end{tabular}

Table 4: Effects table for the replicated height

\begin{tabular}{|l|l|}
\hline Effect & Approximated \\
\hline Average & 267.8 \\
\hline A:Tmelt & 240.8 \\
\hline B:Tmould & 275.7 \\
\hline C:Filling time & -119.9 \\
\hline D:Cavity length & 478.2 \\
\hline AB & 134.1 \\
\hline AC & -93.6 \\
\hline AD & 240.8 \\
\hline BC & -79.9 \\
\hline BD & 223.2 \\
\hline CD & -117.4 \\
\hline
\end{tabular}

is the surface-to-volume ratio leads to an increase of the heat transfer and, hence, to the faster solidification of the polymer (Piotter[17], Chen [18]).

We see that both the temperature of the mould and the temperature of the melt are factors that help similarly the replication of the marks. This is a logic result since the marks are copied until the polymer reaches the glass transition temperature, hence, the bigger the mould and melt temperatures, the bigger the time the polymer has to go into the mark before reaching its glass transition temperature. Besides that, the bigger the temperature, the less viscous the polymer is and the easier it goes into the marks. This results are in accordance with experimental works carried out by Kari Mönkkönen [19], Tofteberg and Andreassen [8], Chen [18] and Sha [20]. From an industrial point of view, an increase of these temperatures lead to a more expensive process due to (i) more time is needed for cooling the injected part to the ejection temperature and (ii) the larger energy consumption needed for warming both the mould and the polymer. Concerning to the range where the mould and wall temperatures should be 
modified, Stormonth [21] comments that the changes in the mould and wall temperature has a bigger effect when set around the optimal temperatures suggested when using conventional injection moulding.

Hattori [22] shows an interesting experimental approach consisting in using a thin-film heater used for heating the mould surface in the nanostructured region, this, in concordance with our simulation results, leads to an improvement of the cavities replication. Rytka [15] concluces that variothermal injection moulding can replicate with high accuracy high aspect ratio microstructures when combinated with a polymer of low melt viscosity.

Related to the filling time, it is seen that the increase of the filling time leads to a fewer replication of the mark, if we look at the pressure profile of the cavities we obtain the plot of the figure 14 .

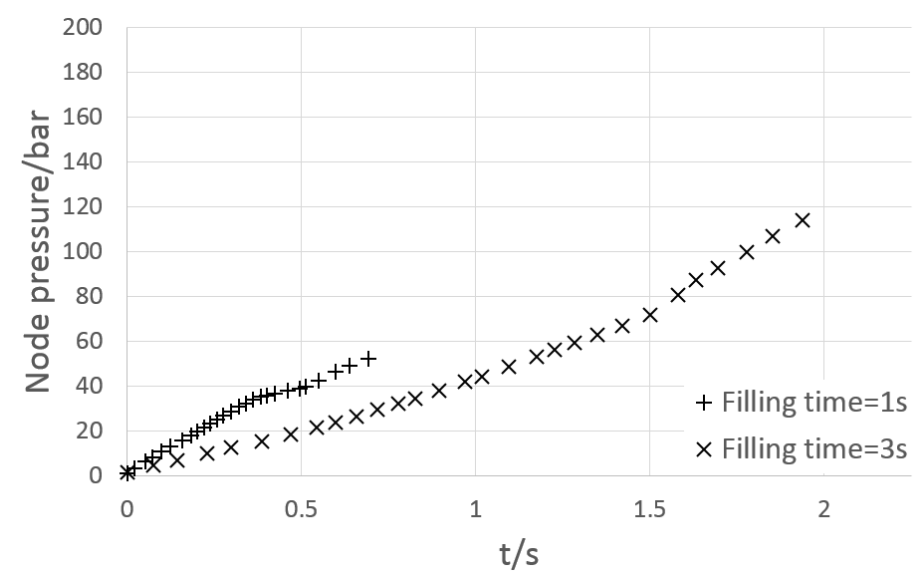

Figure 14: Filling time vs. pressure, data from the macrosimulation. It is observed that, even though the 3 s filling time leads to a bigger pressure at advanced times, the 1s filling time leads to a bigger pressure in the beginning of the injection, when the polymer is still fluid. This is the reason for the negative effect of the filling time factor.

We see clearly that with larger filling times, the pressure that reaches the cavitiy during the time before freezing (which is for all the polymers fewer than the $0.692 \mathrm{~s}$ ) decreases. The positive effect of the pressure in the replication has already been addressed in the literature by Chen [18] and Pranov [23]. From an industrial point of view it is concluded therefore, that for improving the replication of cavities larger injection pressures are needed, which are obtained only with injection machines with large hydraulic pressures available.

Related to the effect of the holding pressure, from a fluid dynamics point of view, the holding pressure has no influence in the cavities replication. Reason is that the holding pressure is applied after the filling stage, and by then, the polymer is already a solid inside the cavities due to the large surface-to-volume ratio. However, divergences are found in the literature when reading experimental papers. Sha [20] shows experimentally that the holding pressure could slightly help to improve the replication, however, for PP and ABS a decrease of the filling length of microcavities was observed. Tofteberg [24] experimentally shows that the holding pressure is an effecting factor, like Sha [20] work, Tofteberg experiments show that sometimes the holding pressure has a positive effect and in other experiments has a negative effect. Chen [18] experimentally studied the effect of the holding pressure and shows a positive effect of the holding pressure in the depth of the microchannel and a negative effect of the holding pressure in the width of the microchannel, though of less importance than other factors like the mould temperature, the melt temperature and the filling time. Rytka ([15] carried out experiments using compression moulding and found no influence on the replicated structure height using an additional compression stroke, reason is, according to their publication, a rapid formation of a frozen polymer layer due to fast cooling upon contact with the mould, this frozen layer prevents further filling of the micro-cavities in the mould during the compression phase. Rytka exposes [10] that the packing phase is less relevant for mould temperatures below the no-flow temperature, because the polymer in the cavity has cooled down below the no flow temperature after about $0.5 \mathrm{~s}$, what prevents further filling of the cavity once in the packing phase.

An important success of our model is that we don't tune the heat transfer coefficient like the simulation approaches of Tofteberg [8], Gang [25] or Rytka [10]. Instead, we let the CFD solver calculate the fluid-side heat transfer based on the local flow-field conditions (e.g., turbulence level, temperature, and velocity profiles), using the following equation in a laminar flow:

$$
q=k_{f}\left(\frac{\partial T}{\partial n}\right)_{\text {wall }}
$$

where $n$ is the local coordinate normal to the wall and $k_{f}$ the thermal conductivity of the fluid.

\subsection{Experimental results}

The patterns on the injected pieces have been characterized by AFM. The results are shown in figures 15 and 16 for positions G2 and P2 (center of the mould), respectively. The marks placed close to the central hole (G3 and P3) disappear during the removal of the perpendicular stick. The marks placed far from the injection point (G1 and P3) are not much replicated, the reason is that these cavities reach smaller pressures and, as proved in the simulations, the pressure is a factor with a positive effect.

The most striking observation is the few replication of the cavities, the injected patterns exhibit heights below $100 \mathrm{~nm}$, far from the $900 \mathrm{~nm}$ depth from the (negative) mould part.

It is observed that the polymer reaches a larger depth when the marks are bigger (G2 compared to P2). This is a result in good correlation with the simulation results, where it was stated that the cavity length is a factor with a positive effect.

It is observed in the AFM measurements, that the shape and the depth reached by the polymer in the cross 

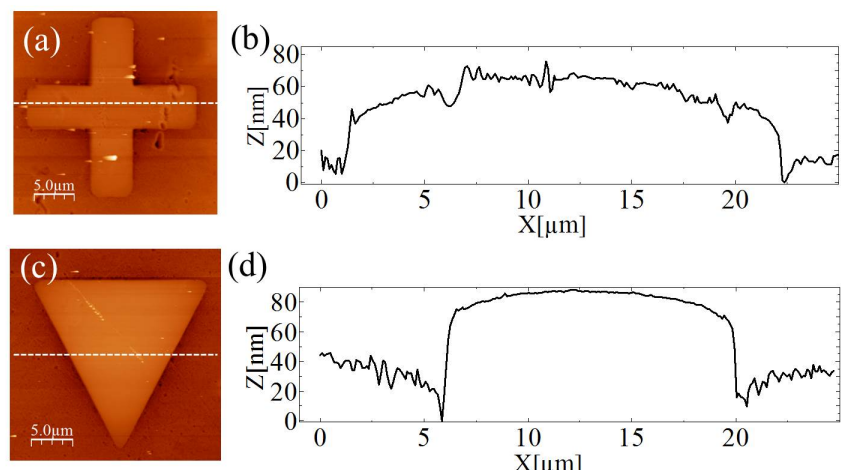

(d)
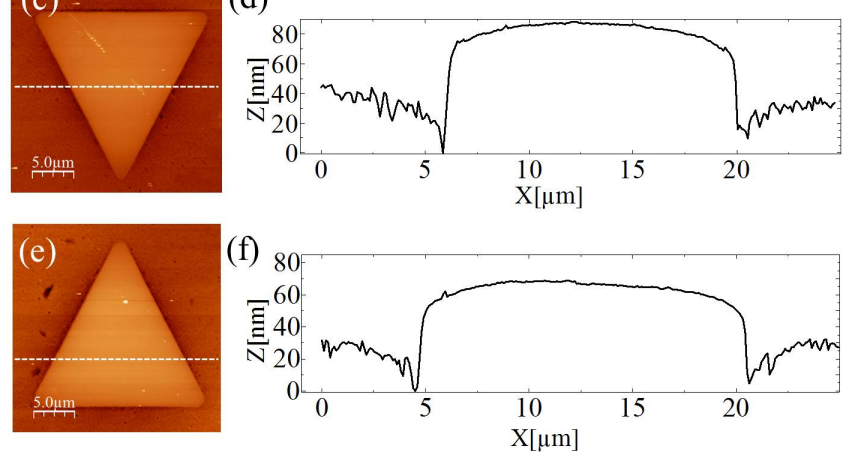

Figure 15: AFM images of the transferred patterns placed at the centre of the mould (G2) and corresponding cross sections.
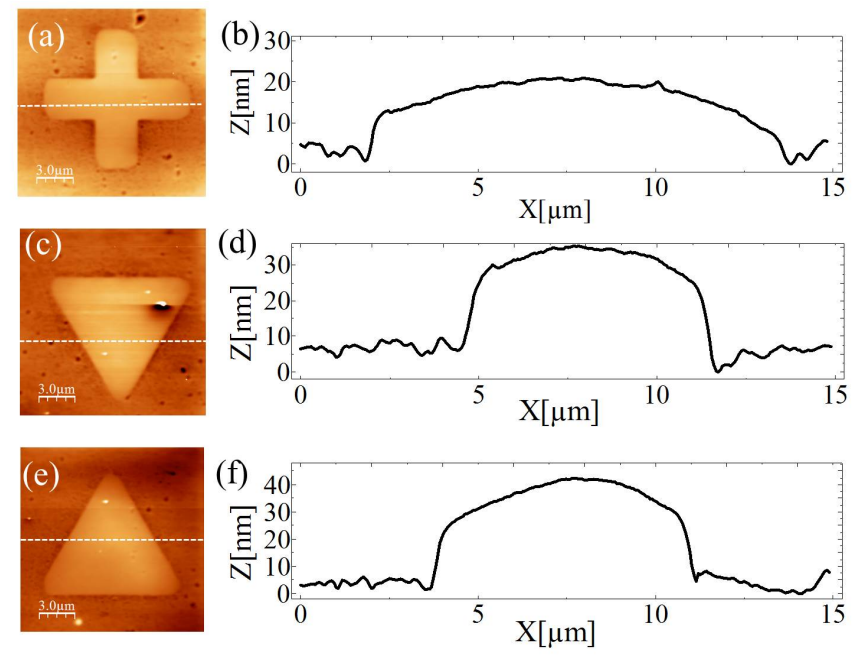

Figure 16: AFM images of the transferred patterns placed at the centre of the mould (P2) and corresponding cross sections.

shaped cavities is the same for both directions: parallel and perpendicular to the polymer flow. This experimental observation reinforces the two stages approach used in the simulation part.

\section{Conclusions}

It is concluded that the submodeling approach is a valid approach for simulating how nanocavities are filled in the injection moulding manufacturing process. Using the design of experiments of the table 3 the main effects are quantified as shown in the Pareto diagram of the figure 12. It is concluded that the cavity length, the temperature of the mould and the temperature of the polymer are positive effects with 5,3 and 2.6 standardized effects values. On the contrary, the filling time is a factor with a negative standardized effect of -1.3 .

Concerning to the experimental part, it is concluded that the FIB technique is able to manufacture cavities at the nanoscale whose measuring using an AFM gives light in understanding how the polymer flows into the marks. Replication of the marks far from the injection point is poor due to the smaller pressure reached in this area, which is a result in good correlation with the simulation part. Cavity length is experimentally observed to be a factor with a positive effect in the replication.

The fact that the shape of the polymer in the cross shaped cavities is the same for both directions (perpendicular and parallel to the flow) reinforces the two stages simulation approach. Future work in the simulation will be the expansion of the algorithm to the three dimensions, this will provide us more know-how related to how the geometry effects the replication of the nanocavities.

\section{Acknowledgments}

This work has been performed within the aim4np project (Automated in-line Metrology for Nanoscale Production), which is supported by the EC through a grant (contract Nr. 309558) within the 7th Frame-work Program NMP Call 2012.1.4-3 on Nanoscale mechanical metrology for industrial processes and products. ICN2 acknowledges support of the Spanish MINECO through the Severo Ochoa Centers of Excellence Program under Grant SEV-20130295 .

\section{References}

[1] Hwajin Oh, Jaehong Park, Youngseok Song, and Jaeryoun Youn. Micro-injection Moulding of Lab-on-a-chip ( LOC ). 19, 2011.

[2] Y. E. Yoo, T. H. Kim, D. S. Choi, S. M. Hyun, H. J. Lee, K. H. Lee, S. K. Kim, B. H. Kim, Y. H. Seo, H. G. Lee, and J. S. Lee. Injection molding of a nanostructured plate and measurement of its surface properties. Current Applied Physics, 9(2 SUPPL.):e12-e18, 2009.

[3] Sohee Kim, Une Teak Jung, Soo Kyoung Kim, Joon Hee Lee, Hak Soo Choi, Chang Seok Kim, and Myung Yung Jeong. Nanostructured multifunctional surface with antireflective and antimicrobial characteristics. ACS Applied Materials and Interfaces, 7(1):326-331, 2015.

[4] Sunandan Baruah, Samir K Pal, and Joydeep Dutta. Nanostructured Zinc Oxide for Water Treatment. Nanoscience and Technology-Asia, 2(2):90-102, 2012.

[5] Alexander B. Christiansen, Jeppe S. Clausen, N. Asger Mortensen, and Anders Kristensen. Injection moulding antireflective nanostructures. Microelectronic Engineering, 121:47$50,2014$.

[6] Adekunle a Fagade and David O Kazmer. Early Cost Estimation for Injection Molded Parts. Journal of Injection Molding Technology, 3(4):97-106, 2000.

[7] J. C T Eijkel and Albert van den Berg. Nanofluidics: What is it and what can we expect from it? Microfluidics and Nanofluidics, 1(3):249-267, 2005.

[8] T Tofteberg and E Andreassen. Injection moulding of microfeatured parts. Proceedings of the Polymer Processing Society 24th Annual Meeting, PPS-24, 2008. 
[9] Liyong Yu, L James Lee, and Kurt W Koelling. Flow and heat transfer simulation of injection molding with microstructures. Polymer Engineering \& Science, 44(10):1866-1876, 2004.

[10] Tim Osswald and Natalie Rudolph. Polymer Rheology. Hanser, 2014.

[11] C G Gal, M Grasselli, and A Miranville. Cahn-Hilliard-NavierStokes systems with moving contact lines. Calculus of Variations and Partial Differential Equations, 55(3):1-47, 2016.

[12] Sascha Kuhn, August Burr, Michael Kübler, Matthias Deckert, and Christoph Bleesen. Study on the replication quality of micro-structures in the injection molding process with dynamical tool tempering systems. Microsystem Technologies, 16(10):1787-1801, 2010.

[13] a.G Fallis. Optimizing the Filling Time and Gate of the Injection Mold on Plastic Air Intake Manifold of Engines. Journal of Chemical Information and Modeling, 53(9):1689-1699, 2013.

[14] C Rytka, P M Kristiansen, and A Neyer. Iso- and variothermal injection compression moulding of polymer micro- and nanostructures for optical and medical applications, 2015.

[15] G A Mannella, V La Carrubba, V Brucato, W Zoetelief, and G Haagh. No-flow temperature in injection molding simulation. Journal of Applied Polymer Science, 119(6):3382-3392, 2011.

[16] V. Piotter, K. Mueller, K. Plewa, R. Ruprecht, and J. Hausselt. Performance and simulation of thermoplastic micro injection molding. Microsystem Technologies, 8(6):387-390, 2002.

[17] Chun Sheng Chen, Shia Chung Chen, Won Hsion Liao, Rean Der Chien, and Su Hsia Lin. Micro injection molding of a micro-fluidic platform. International Communications in Heat and Mass Transfer, 37(9):1290-1294, 2010.

[18] Kari Monkkonen, Tuula T. Pakkanen, Joni Hietala, Esko J. Pakkonen, Pertti Pakkonen, Timo Jskelinen, and Terho Kaikuranta. Replication of sub-micron features using amorphous thermoplastics. Polymer Engineering and Science, 42(7):1600-1608, 2002.

[19] Baichuan Sha, Stefan Dimov, Christian Griffiths, and Michael S. Packianather. Micro-injection moulding: Factors affecting the achievable aspect ratios. International Journal of Advanced Manufacturing Technology, 33(1-2):147-156, 2007.

[20] John Moir Stormonth-Darling. Fabrication of difficult nanostructures by injection moulding. pages 1-157, 2013.

[21] Shuntaro Hattori, Keisuke Nagato, Tetsuya Hamaguchi, and Masayuki Nakao. Rapid injection molding of high-aspect-ratio nanostructures. Microelectronic Engineering, 87(5-8):15461549, 2010.

[22] Henrik Pranov, Henrik Koblitz Rasmussen, Niels Bent Larsen, and Nikolaj Gadegaard. On the injection molding of nanostructured polymer surfaces. Polymer Engineering and Science, 46(2):160-171, 2006.

[23] T R Tofteberg, H Amédro, F Grytten, and E Andreassen. Effects of Injection Molding Holding Pressure on the Replication of Surface Microfeatures. International Polymer Processing, 25(3):236-241, jul 2010.

[24] C Rytka, J Lungershausen, P M Kristiansen, and A Neyer. 3D filling simulation of micro- and nanostructures in comparison to iso- and variothermal injection moulding trials. Journal of Micromechanics and Microengineering, 26(6):65018, 2016.

[25] Won Woo Kim, Myeong Gu Gang, Byung Kwon Min, and Wook Bae Kim. Experimental and numerical investigations of cavity filling process in injection moulding for microcantilever structures. The International Journal of Advanced Manufacturing Technology, pages 293-304, 2014. 